\title{
Long Non-Coding RNA RP11-789C1.1 Suppresses Epithelial to Mesenchymal Transition in Gastric Cancer Through the RP11-789C1.1/MiR-5003/E-Cadherin Axis
}

\author{
Zehong Chen ${ }^{a}$ Jialin $\mathrm{Wu}^{\mathrm{a}} \quad$ Wensheng Huang ${ }^{\mathrm{a}}$ Jianjun Peng ${ }^{\mathrm{a}}$ \\ Jinning Ye $\mathrm{e}^{\mathrm{a}}$ Liang Yang ${ }^{\mathrm{a}}$ YuJie Yuan ${ }^{\mathrm{a}}$ Chuangqi Chen ${ }^{\mathrm{a}}$ \\ Changhua Zhang $^{\mathrm{a}}$ Shirong Cai ${ }^{a}$ Yulong He ${ }^{a}$ Suijing Wu ${ }^{b}$ Wu Song ${ }^{a}$ \\ aDepartment of Gastrointestinal Surgery, The First Affiliated Hospital, Sun Yat-sen University, \\ Guangzhou, bDepartment of Hematology, Guangdong General Hospital, Guangdong Academy of \\ Medical Sciences, Guangzhou, China
}

\section{Key Words}

Gastric cancer $\bullet$ LncRNA • MiRNA • E-cadherin • EMT

\begin{abstract}
Background/Aims: Gastric cancer (GC) is a common malignancy with a global incidence that ranks fourth among all tumor types. Epithelial-to-mesenchymal transition (EMT) is a tumor biological process with a role in GC cell metastasis. Long non-coding RNAs (IncRNAs) and microRNAs possess important regulatory functions at the cellular level and in diverse pathophysiological processes. This study was conducted to investigate whether IncRNA RP11789C1.1 regulates EMT in GC by mediating the miR-5003/E-cadherin pathway. Methods: RP11789C1.1 and miR-5003 expression was detected in GC specimens and cell lines by quantitative real-time PCR. Western blotting and immunohistochemistry were performed to detect EMT markers in GC. Cell Counting Kit 8 assays were carried out to explore cell proliferation. Wound healing and Transwell assays were conducted to determine the migration and invasion of GC cells. To clarify the correlation between RP11-789C1.1, miR-5003, and E-cadherin, dualluciferase reporter assays were applied. Results: LncRNA RP11-789C1.1 was significantly down-regulated in GC patients and cell lines, along with the concomitant up-regulation of miR-5003. Silencing RP11-789C1.1 and over-expressing miR-5003 significantly promoted the tumor behavior of GC cells. Dual-luciferase reporter assays confirmed that miR-5003 was the target of both RP11-789C1.1 and E-cadherin. Furthermore, at both the mRNA and protein level, silencing RP11-789C1.1 remarkably reduced the expression of E-cadherin and promoted EMT, which were reversed by knocking down miR-5003. Conclusions: LncRNA RP11-789C1.1 inhibited EMT in GC through the RP11-789C1.1/miR-5003/E-cadherin axis, which could be a promising therapeutic target for GC.
\end{abstract}

Z. Chen, J Wu and W. Huang contributed equally to this work. 


\section{Introduction}

Gastric cancer is one of the most common malignancies and the second leading cause of cancer-related death worldwide, with a global incidence that ranks fourth among all tumor types $[1,2]$. Over the past few decades, despite numerous efforts to improve the survival of patients with gastric cancer, such as improved living standards, a reduction in chronic Helicobacter pylori infections, and remarkable advances in diagnosis and treatment, the 5 -year overall survival rate of patients with gastric cancer is still lower than $30 \%$, due to the late advanced stage at which it is diagnosed and the high incidence of tumor metastasis and recurrence $[3,4]$. Therefore, there are still considerable clinical challenges regarding how to detect this malignancy early and to improve its prognosis. It is of great importance to identify novel prognostic biomarkers and to elucidate the underlying molecular mechanisms concerning the initiation, development, and metastasis of gastric cancer, and furthermore, to explore additional effective therapeutic approaches for patients with gastric cancer.

Recently, an increasing number of studies has revealed that non-coding RNAs such as microRNAs (miRNAs) and long non-coding RNAs (lncRNAs) play critical roles in the development of cancer [5-7]. LncRNAs are more than 200 nucleotides in length with no or weak protein coding ability, while microRNAs consist of approximately 22 nucleotides $[8,9]$. LncRNAs and miRNAs individually or jointly regulate diverse biological processes, including the immune response, cell apoptosis, differentiation, development, cancer cell metastasis, and drug resistance [10-13]. Several studies have reported that IncRNAs can promote development and metastasis in various types of cancer by acting as a competing endogenous RNA (ceRNA) "sponge" for miRNAs or vice versa [14]. For example, it has been shown that IncRNA RoR can induce epithelial-to-mesenchymal transition (EMT) to promote breast cancer progression and metastasis by acting as a ceRNA for miRNA (miR)-205 [15], while hsa-miR-125b suppresses bladder cancer progression by negatively regulating lncRNA metastasis-associated lung adenocarcinoma transcript 1 [16]. Hence, identifying the interactions between lncRNAs and miRNAs may help to provide new aspects for cancer diagnosis and treatment.

During EMT, epithelial cells lose their polarity and transit into a mesenchymal phenotype, and this process has been shown to have a role in cancer cell metastasis [17]. The most important molecular markers of EMT are considered to be E-cadherin, N-cadherin, and vimentin [18]. Transcription factors such as Snail, Slug, and ZEB1 have been shown to inhibit E-cadherin expression [19]. Previously, we found that IncRNA XLOC_010235 was upregulated in gastric cancer tissues and demonstrated that it was an effective biomarker for cancer EMT by targeting the EMT inducer Snail1 [20].

Another significantly dysregulated IncRNA detected in our IncRNA expression microarray, RP11-789C1.1, was verified to be down-regulated in gastric cancer cells and closely associated with tumor metastasis and patient prognosis. In the present study, we investigated the effects of RP11-789C1.1 expression on gastric cancer cell phenotypes in vitro, and found that the abnormal expression of RP11-789C1.1 can significantly affect the expression of multiple EMT-related molecules, including E-cadherin, $\mathrm{N}$-cadherin, and vimentin, by binding directly to a mediator, miR-5003-3p, which was verified to promote cancer by targeting E-cadherin, suggesting that RP11-789C1.1 affects the invasion and metastasis of gastric cancer cells through the miR-5003-EMT cascade. Taken together, these results suggest that IncRNA RP11-789C1.1, considered to be a tumor suppressor, functions as a ceRNA sponge for miRNA-5003-3p and further plays a significant role in the progression and metastasis of gastric cancer via EMT, which indicates that it may serve as a marker for gastric cancer metastasis.

\section{Materials and Methods}

Cell lines and culture

Human esophageal cancer cell lines (AGS, BGC-803, HGC-27, SGC-7901, MKN-1, MKN28, and MGC803) were obtained from the American Type Culture Collection (Manassas, VA, USA). The normal human gastric 


\section{Cellular Physiology Cell Physiol Biochem 2018;47:2432-2444 \\ \begin{tabular}{l|l} 
DOI: 10.1159/000491617 & $\begin{array}{l}\text { O } 2018 \text { The Author(s). Published by S. Karger AG, Basel } \\
\text { www.karger.com/cpb }\end{array}$
\end{tabular} \\ Chen et al.: LncRNA RP11-789C1.1 Regulates EMT Through RP11-789C1.1/MiR-5003/E- \\ Cadherin Axis}

epithelial cell line GES-1 was purchased from the Shanghai Cell Bank of the Chinese Academy of Science (Shanghai, China). AGS, BGC-803, HGC-27, MKN-1, MKN28, and MGC803 cells were cultured in RPMI 1640 (Gibco, Carlsbad, CA, USA), while SGC-7901 and GES-1 cells were cultivated in Dulbecco's modified Eagle's medium (DMEM; Gibco) supplemented with 10\% fetal bovine serum (Gibco) and 0.1\% penicillin/ streptomycin (Invitrogen, Carlsbad, CA, USA). The cells were incubated and maintained in a humidified atmosphere of $5 \% \mathrm{CO}_{2}$ at $37^{\circ} \mathrm{C}$.

\section{RNA extraction and quantitative real-time reverse transcription PCR analysis}

Total RNA was isolated from clinical specimens and cell lines using the TRIzol reagent (Invitrogen, Carlsbad, CA, USA). Real-time quantitative reverse transcription PCR (qRT-PCR) was performed with a standard SYBR Green PCR Kit (TaKaRa, Dalian, China) on a LightCycler 480 platform (Roche, Meylan, France). The expression of mRNAs and IncRNAs was normalized to the levels of glyceraldehyde-3-phosphate dehydrogenase (GAPDH), while miRNAs were normalized to U6. The specific primers for mRNAs are presented in Table 1 . The data were collected and analyzed using the $2^{-\Delta \Delta C t}$ method.

\section{Cell transfection}

A riboFECT CP Transfection Kit (RiboBio, Guangzhou, China) was used for transfection according to the product description. RP11-789C1.1 small interfering RNA (siRNA-RP) and scrambled siRNA (siRNANC), as well as pcDNA3.1-RP11-789C1.1 (pcDNA-RP) and pcDNA3.1-control (pcDNA-NC) were obtained from iGeneBio (Guangzhou, China). miRNA-5003-3p mimic (miR-5003), miR-5003-3p mimic control (miRNC), miR-5003-3p inhibitor (in-miR-5003), and inhibitor control (in-miR-NC) were purchased from RiboBio (Guangzhou, China).

\section{Cell proliferation assay}

Cell viability was measured using a Cell Counting Kit 8 (CCK8) (Dojindo, Kumamoto, Japan). The cells were transfected with miRNA, siRNA, or pcDNA3.1 and grown on 96-well plates. Cell viability was monitored every $24 \mathrm{~h}$ according to the manufacturer's protocol.

\section{Wound-healing assay}

Cells $\left(3.0 \times 10^{5}\right)$ transfected with miRNA, siRNA, or pcDNA3.1 were plated on 6-well plates and cultured for monolayer formation. The cell layer was scratched with a sterile plastic tip. After washing with phosphate-buffered saline 3 times, the cells were cultured in DMEM (Gibco) or RPMI 1640 supplemented with $1 \%$ fetal bovine serum (Gibco). Images of the plates were captured under a microscope at 0 and 48 $\mathrm{h}$ after scratching. Digimizer image analysis software was used to measure the distance between the two edges of the scratch.

\section{Cell migration and invasion assays}

Transwell inserts with 8- $\mu$ m pore size polycarbonate (Corning, Inc., Corning, NY, USA) were applied for cell migration and invasion assays. For the migration assays, $5.0 \times 10^{4}$ cells in serum-free medium were placed in the upper chamber. For the invasion assays, $5.0 \times 10^{4}$ cells in serum-free medium were placed in the upper chamber, which was coated with Matrigel (BD Biosciences, Franklin Lakes, NJ, USA). DMEM or RPMI 1640 with $10 \%$ fetal bovine serum was added to the lower chamber. The cells were incubated for $48 \mathrm{~h}$. The cells on the lower membrane surface were considered to be migrated or invaded cells. These cells were fixed with methanol for $20 \mathrm{~min}$ and stained with $0.1 \%$ crystal violet. Images were captured under a microscope and the number of migrated or invaded cells was counted.

\section{Western blot analysis}

The cells were harvested and total protein was extracted using RIPA protein extraction reagent (Beyotime, Beijing, China) supplemented with phenylmethylsulfonyl fluoride (Roche, Pleasanton,

Table 1. Specific primers and siRNA sequences used in the present study

\begin{tabular}{|c|c|c|}
\hline Name & Primer or siRNA $\mathrm{s}$ & equence $\left(5^{\prime}-3^{\prime}\right)$ \\
\hline & Forward & Reverse \\
\hline RP11-789C1.1 & ACAAGACCAGGAGGAGGTTT & GTAGTTGCTCTCCTGCTTGTATG \\
\hline E-cadherin & TGCCTGAGAACGAGGCTAAC & TGGGGGCTTCATTCACATCC \\
\hline $\mathrm{N}$-cadherin & TCTGGGTCTGTTTTATTACTCCTGG & CGAGCTGATGACAAATAGCGG \\
\hline Vimentin & AACTTAGGGGCGCTCTTGTC & CCTGCTGTCCCGCCG \\
\hline MMP-2 & CGTCTGTCCCAGGATGACATC & TGTCAGGAGAGGCCCCATAG \\
\hline MMP-9 & TGGGCAGATTCCAAACCTTT & TCTTCCGAGTAGTTTTGGATCCA \\
\hline GAPDH & GСтCTCTGCTCCTCCTGTTC & ACGACCAAATCCGTTGACTC \\
\hline siRNA-RP11-789C1.1-1 & \multicolumn{2}{|c|}{ GATTGGCATGTGATCACTT } \\
\hline siRNA-RP11-789C1.1-2 & \multicolumn{2}{|c|}{ CCTGAGTTGCAGGTCCATA } \\
\hline siRNA-RP11-789C1.1-3 & \multicolumn{2}{|c|}{ GCACGGACTATTCTCTCAT } \\
\hline
\end{tabular}




\section{Cellular Physiology Cell Physiol Biochem 2018;47:2432-2444 \begin{tabular}{l|l} 
DOI: 10.1159/000491617 & Ond Biochemistry \\
Published online: July 09, 2018 & $\begin{array}{l}\text { 2018 The Author(s). Published by S. Karger AG, Basel } \\
\text { www.karger.com/cpb }\end{array}$
\end{tabular} \\ Chen et al.: LncRNA RP11-789C1.1 Regulates EMT Through RP11-789C1.1/MiR-5003/E- \\ Cadherin Axis}

CA, USA). Protein concentrations were determined using a BCA Protein Assay Kit (Beyotime). A total of $50 \mu \mathrm{g}$ protein was separated using $8-12 \%$ sodium dodecyl sulfate-polyacrylamide gel electrophoresis and then transferred to a polyvinylidene fluoride membrane (Millipore, Billerica, MA, USA). The membrane was blocked with 5\% non-fat milk and incubated with primary antibodies against GAPDH (rabbit monoclonal), E-cadherin, and N-cadherin (1:10000; Abcam, Cambridge, UK), and vimentin, MMP-2, and MMP-9 (1:1000; Cell Signaling, Danvers, MA, USA) overnight at $4^{\circ} \mathrm{C}$. Then, the membrane was incubated with a secondary antibody (1:5000; Biogot Technology, Nanjing, China) for $2 \mathrm{~h}$ at room temperature. Enhanced chemiluminescence substrate (Millipore) was used to visualize the protein bands.

\section{Immunohistochemical staining}

Paraformaldehyde-fixed paraffin-embedded sections were dewaxed and dehydrated. After rehydration and antigen retrieval with a citrate buffer, endogenous peroxidase activity was blocked by $\mathrm{H}_{2} \mathrm{O}_{2}(3.0 \%)$. The sections were blocked with $10 \%$ goat plasma for $30 \mathrm{~min}$. Primary antibodies against E-cadherin (1:400; Abcam) were added to the sections and incubated at $4{ }^{\circ} \mathrm{C}$ overnight. Detection of the primary antibodies was performed using biotinylated secondary antibodies. Hematoxylin was used for counterstaining.

\section{RNA pull-down assay}

For pull-down assays of biotinylated miR-5003, miR-5003-mut and control were synthesized and transfected into MKN-1 and SGC-7901 cells. The cells were then collected and lysed. According to the product description, a portion of extract was used for input and another portion was incubated with Dynabeads M-280 Streptavidin (Invitrogen). RNA was purified with an RNA Isolation Kit (QIAGEN, Valencia, CA, USA) and used for qRT-PCR assays.

For pull-down assays of the biotinylated DNA probe, the complementary and biotinylated probe of RP11-789C1.1 was obtained and Dynabeads M-280 Streptavidin (Invitrogen) was used to generate probecoated beads. The probe-coated beads were incubated with lysates from MKN-1 and SGC-7901 cells. The binding RNAs were then collected and used for qRT-PCR.

\section{Dual luciferase reporter assay}

The binding sites between miR-5003-3p and RP11-789C1.1 or E-cadherin were predicted by bioinformatics tools (microRNA.org, Starbase v2.0, and miRcode). Dual luciferase reporters were constructed according to the procedures described in our previous study [21]. A Dual Luciferase Reporter Assay System (Promega, Madison, WI, USA) was applied to measure luciferase activity. Renilla luciferase activity was used for normalization. The experiments were repeated independently 3 times.

\section{Statistical analysis}

Data are presented as the mean \pm standard error of the mean. All statistical analyses were performed using SPSS 19.0 statistical software (SPSS, Inc., Chicago, IL, USA). Student's t-test (2-tailed), one-way analysis of variance, and the Mann-Whitney $U$ test were used for statistical analyses. P-values less than 0.05 were considered statistically significant.

\section{Results}

LncRNA RP11-789C1.1 suppresses the proliferation of gastric cancer cells

We previously demonstrated that lncRNA RP11-789C1.1 was significantly downregulated in gastric cancer specimens and was closely associated with patient prognosis. Therefore, we sought to further evaluate the biological role of RP11-789C1.1 in gastric cancer cells. Compared with the normal gastric epithelial cell line GES-1, RP11-789C1.1 was found to be remarkably down-regulated in all 7 gastric cancer cell lines examined (Fig. 1A). We then randomly selected 2 gastric cancer cell lines for over-expression or knockdown assays. pcDNA-RP was transfected into MKN-1 cells for the over-expression of RP11-789C1.1, and siRNA-RP was transfected into SGC-7901 cells for the knockdown of RP11-789C1.1. pcDNA-NC or siRNA-NC was also transfected into these cells as a respective control group. Transfection efficiency was validated by qRT-PCR (Fig. 1B). CCK8 assays were conducted to 
explore the impact of RP11-789C1.1 on gastric cancer cells. The results indicated that RP11789C1.1 over-expression significantly reduced the proliferation of MKN-1 cells, while RP11789C1.1 knockdown remarkably enhanced the proliferation of SGC-7901 cells (Fig. 1C). These results demonstrated that lncRNA RP11-789C1.1 could suppress the proliferation of gastric cancer cells.

LncRNA RP11-789C1.1 suppresses the migration, invasion, and EMT of gastric cancer cells

To further determine whether RP11-789C1.1 could have an impact on the progression of gastric cancer, we explored its effect on the migration and invasion of MKN-1 and SGC7901 cells. Using a wound healing assay, we found that MKN-1 cells transfected with pcDNARP had a slower scratch closure rate than the control group. SGC-7901 cells transfected with siRNA-RP had a faster scratch closure rate than the control group (Fig. 1D). Using Transwell migration assays (without Matrigel) and Transwell invasion assays (with Matrigel), we found that the mobility and invasiveness of MKN-1 cells were dramatically suppressed following RP11-789C1.1 over-expression. In parallel, the reduced expression of RP11-789C1.1 remarkably promoted the migration and invasion of SGC-7901 cells when compared with the control group (Fig. 1E). Moreover, the results of functional experiments with GES-1 cells were consistent with the results in gastric cancer cells (for all online suppl. material, see www.karger.com/doi/10.1159/000491617, Fig. S1A). Thus, we concluded that lncRNA RP11-789C1.1 could significantly inhibit the migration and invasion of gastric cancer cells. EMT is associated with the progression and metastasis of gastric cancer cells; therefore, we explored whether RP11-789C1.1 had any influence on the EMT of gastric cancer cells. Immunohistochemistry was firstly performed to explore the association between RP11-

Fig. 1. Effects of IncRNA RP11-789C1.1 on gastric cancer cell proliferation, migration, and invasion in vitro. A. RP11-789C1.1 expression in 7 gastric cancer cell lines compared with the normal gastric epithelial cell line GES-1. B. RP11789C1.1 expression in MKN-1 and SGC-7901 cells after transfection with pcDNA-RP and siRNA-RP, respectively. C. CCK8 proliferation assays using MKN-1 and SGC-7901 cells. D. Wound healing assays were performed to investigate migratory ability after transfection. E. Transwell assays were used to investigate the changes in the migratory and invasive abilities of gastric cancer cells after transfection. All experiments were performed in triplicate. ${ }^{*} \mathrm{P}<0.05 ;{ }^{* *} \mathrm{P}<0.01$.

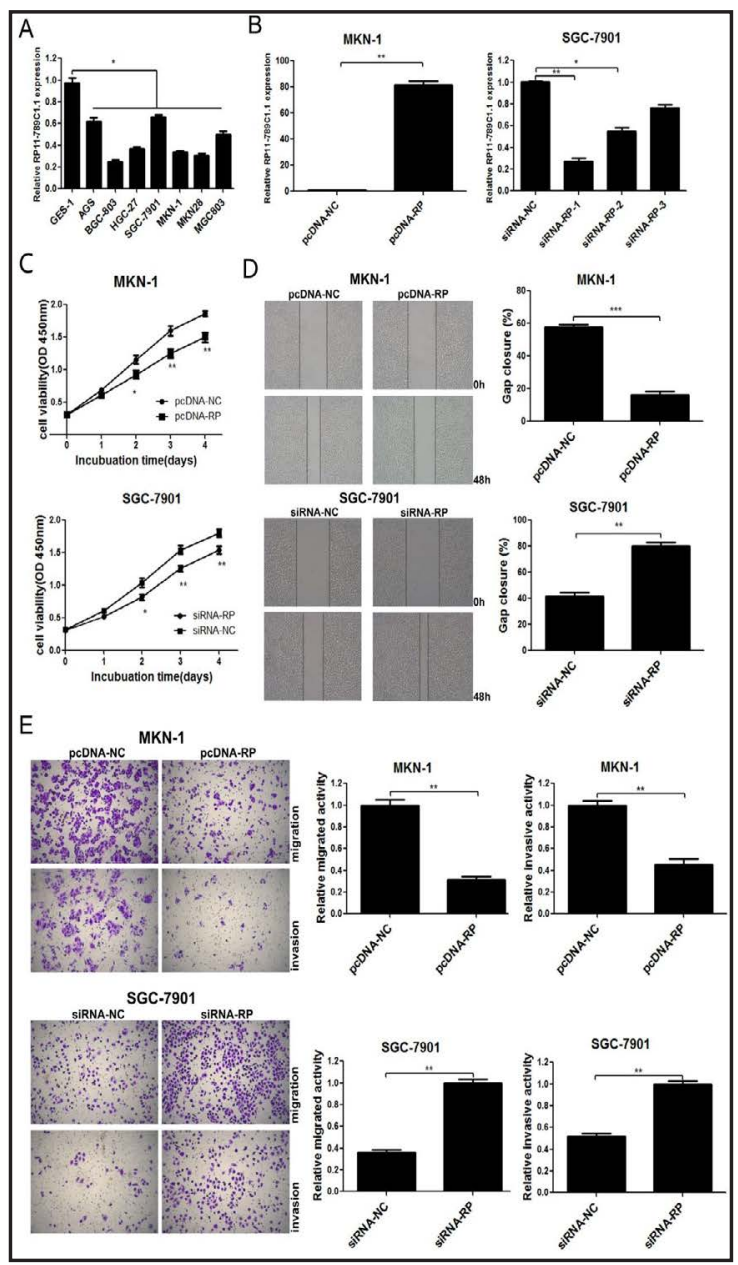


789C1.1 and the EMT markers E-cadherin and N-cadherin in 30 samples from patients with gastric cancer. The patients were divided into high and low RP11-789C1.1 expression groups according to the median expression of RP11-789C1.1 in the total cohort. Compared with low RP11-789C1.1 tissues, E-cadherin expression was significantly increased, while $\mathrm{N}$-cadherin/vimentin expression was decreased in high RP11-789C1.1 tissues (Figs. 2A and see online suppl. material, S1B). These results indicated the potential regulation of EMT by RP11-789C1.1 in gastric cancer. In addition, EMT markers (E-cadherin, N-cadherin, vimentin, MMP-2, and MMP-9) were analyzed in gastric cancer cells following the overexpression or knockdown of RP11-789C1.1. qRT-PCR analysis revealed that RP11-789C1.1 over-expression significantly enhanced the expression of E-cadherin, while it reduced the expression of N-cadherin, vimentin, MMP-2, and MMP-9. Conversely, knockdown of RP11789C1.1 decreased E-cadherin expression, while it increased the expression of $\mathrm{N}$-cadherin, vimentin, MMP-2, and MMP-9 (Fig. 2B, C). Consistently, western blot analysis demonstrated that enhanced RP11-789C1.1 expression could remarkably elevate the expression of E-cadherin and reduce the expression of $\mathrm{N}$-cadherin, vimentin, and Snail, while silencing RP11-789C1.1 generated the opposite expression pattern (Fig. 2D). Thus, these results indicated that RP11-789C1.1 reversely mediated EMT in gastric cancer cells.

\section{LncRNA RP11-789C1.1 directly targets miR-5003-3p in gastric cancer}

To uncover the underlying molecular mechanism by which RP11-789C1.1 influences the progression of gastric cancer, the online bioinformatics tool FINDTAR3 (https://bio. sz.tsinghua.edu.cn) was applied to identify potential targets of RP11-789C1.1. Bioinformatics analysis indicated that miR-5003-3p was a potential target of RP11-789C1.1, which contains a putative 9 base-pair binding site (Fig. 3A). We then used qRT-PCR to assess the expression of miR-5003-3p in 30 paired gastric cancer tissues, consisting of gastric cancer and adjacent normal tissues. miR-5003-3p was found to be dramatically up-regulated in gastric cancer tissues when compared with adjacent normal tissues (Fig. 3B). We also explored the expression pattern of miR-5003-3p and RP11-789C1.1 in these gastric cancer specimens by qRT-PCR. The data showed there was a negative association between miR-5003-3p and RP11-789C1.1 in gastric cancer specimens (Fig. 3C). In addition, we examined miR-5003-3p expression in the GES-1, MKN-1, and SGC-7901 cell lines. miR-5003-3p was found to be upregulated in MKN-1 and SGC-7901 cells when compared with GES-1 cells. Next, miR-5003-3p inhibitors or mimics were transfected into MKN-1 and SGC-7901 cells. Transfection efficiency

Fig. 2. Negative correlation between RP11-789C1.1 and E-cadherin expression, and the effects of RP11789C1.1 on EMT. A. Immunohistochemistry was performed to explore the relationship between RP11-789C1.1 and E-cadherin. E-cadherin immunostaining was positive in gastric cancer tissues with relatively high RP11-789C1.1 expression, while E-cadherin immunostaining was relatively low in gastric cancer tissues with low RP11-789C1.1 expression. B. Relative mRNA levels of EMT pathway markers in $\mathrm{MKN}-1$ cells after RP11-789C1.1 over-expression were analyzed by qRT-PCR. C. Relative mRNA levels of EMT pathway markers in SGC-7901 cells after RP11789C1.1 knockdown. D. Western blot analysis of EMT markers in MKN-1 or SGC-7091 cells with over-expression or knockdown of RP11-789C1.1. ${ }^{*} \mathrm{P}<0.05 ;{ }^{* *} \mathrm{P}<0.01$.

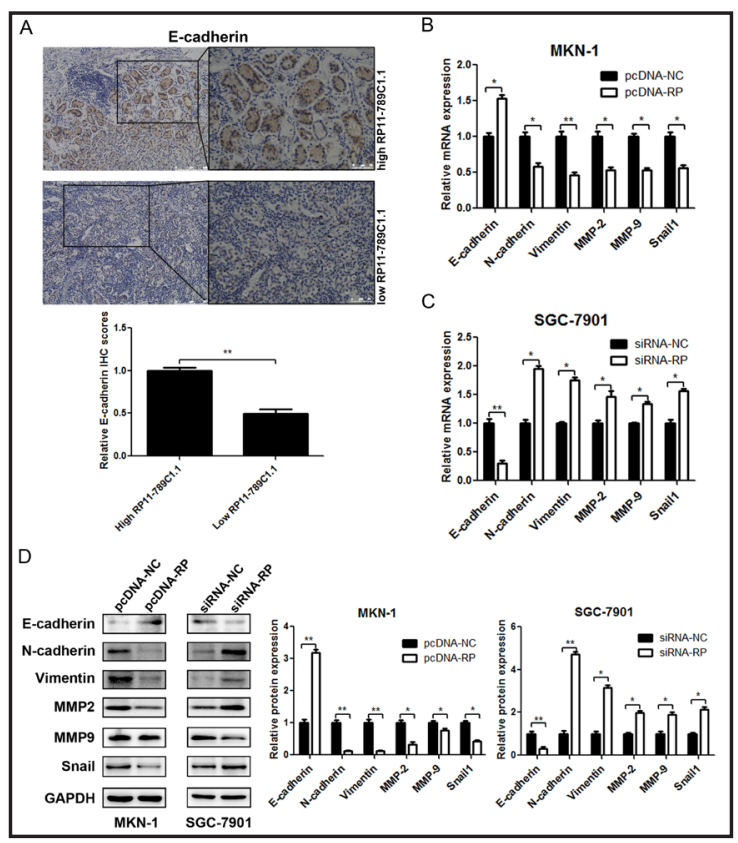


Fig. 3. $m i R-5003-3 p$ is a direct target of RP11-789C1.1 in gastric cancer. A. Bioinformatics analysis showed that miR-5003 could directly target the wild-type $3^{\prime}$ UTR of RP11-789C1.1 (wt-RP). RP11789C1.1-mutant indicates mutation of the binding sites in the $3^{\prime}$ UTR of RP11-789C1.1. B. miR-5003 expression was significantly higher in tumor tissues than in adjacent non-tumor tissues. C. Pearson correlation analysis revealed the clear negative association between miR-5003 and RP11-789C1.1 expression in gastric cancer tissues. D. The relative expression of miR5003 in GES-1, MKN-1, and SGC7901 cells was detected by qRTPCR. The efficiency of miR-5003 knockdown and over-expression was confirmed in MKN-1 and SGC7901 cells. E. Luciferase reporter assays were conducted to verify the binding of RP11-789C1.1 to miR-5003. F. qRT-PCR analysis showed that RP11-789C1.1 could

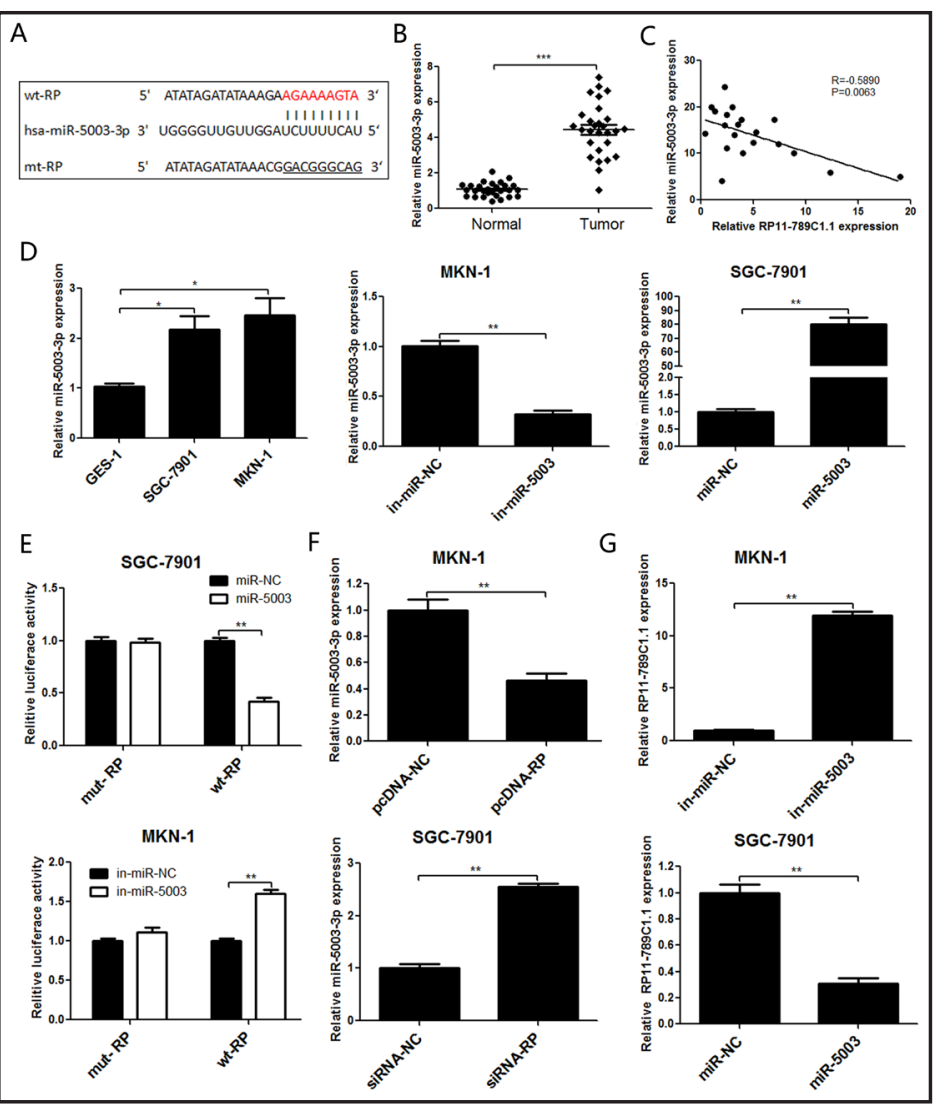
negatively regulate miR-5003 expression in gastric cancer cells. G. miR-5003 inversely regulates RP11-789C1.1 expression in gastric cancer cells. ${ }^{*} \mathrm{P}<0.05 ;{ }^{* *} \mathrm{P}<0.01$.

was validated by qRT-PCR (Fig. 3D). Then, luciferase reporter assays were conducted to verify whether RP11-789C1.1 binds to miR-5003-3p directly. The wild-type 3' untranslated region (UTR) of RP11-789C1.1 (wt-RP) and mutant 3' UTR of RP11-789C1.1 (mut-RP) were cloned into reporter plasmids, respectively. miR-5003-3p was found to reduce the luciferase activity of wt-RP significantly; however, this reduction was not observed using mut-RP (Fig. 3E). Biotin-labeled pull-down assays also indicated that RP11-789C1.1 could target miR5003-3p directly (see online suppl. material, Fig. S3). Furthermore, miR-5003-3p expression was examined in MKN-1 cells, which were transfected with pcDNA-RP, and SGC-7901 cells, which were transfected with siRNA-RP. miR-5003-3p expression was found to be negatively regulated by RP11-789C1.1 in MKN-1 and SGC-7901 cells (Fig. 3F). Moreover, qRT-PCR also revealed that RP11-789C1.1 expression could be enhanced or reduced by modulating the expression of miR-5003-3p in MKN-1 and SGC-7901 cells (Fig. 3G). Thus, we concluded that RP11-789C1.1 targeted miR-5003-3p directly in gastric cancer cells.

miR-5003-3p promotes migration, invasion, and EMT by directly targeting E-cadherin in gastric cancer

miR-5003-3p has been shown to be associated with breast cancer metastasis [22], but its role in gastric cancer is still largely elusive. MKN-1 and SGC-7901 cells were transfected with miR-5003-3p inhibitors or mimics and wound healing and Transwell assays were conducted. The wound healing assays demonstrated that MKN-1 cells transfected with miR-5003-3p inhibitors had a slower scratch closure rate than the control group (Fig. 4A). Conversely, SGC7901 cells transfected with miR-5003-3p mimics exhibited a faster scratch closure rate than the control group (Fig. 4B). The Transwell migration assays (without Matrigel) suggested 
that knockdown of miR-5003-3p inhibited the migration of MKN-1 cells, while its overexpression promoted SGC-7901 cell migration (Fig. 4C, D). The Transwell invasion assays (with Matrigel) showed consistent results (Fig. 4C, D). We then explored whether miR-5003$3 p$ had an influence on the EMT of gastric cancer cells by using immunohistochemistry, qRTPCR, and western blot analyses. The association between miR-5003-3p and the EMT marker E-cadherin was validated by immunohistochemistry. E-cadherin expression was found to be remarkably increased in low miR-5003-3p tissues compared with high miR-5003-3p tissues (Fig. 4E). N-cadherin and Vimentin expression were found to be remarkably increased in high miR-5003-3p tissues compared with low miR-5003-3p tissues (see online suppl. material, Fig. S2). qRT-PCR demonstrated that miR-5003-3p over-expression enhanced the expression of N-cadherin, vimentin, MMP-2, and MMP-9, while it decreased the expression of E-cadherin. Conversely, miR-5003-3p knockdown enhanced the expression of E-cadherin and reduced the expression of N-cadherin, vimentin, MMP-2, and MMP-9 (Fig. 5A, B). Western blot analysis generated similar results (Fig. 5C, D). In order to explore the molecular biological mechanisms by which miR-5003-3p promotes migration, invasion, and EMT in gastric cancer, bioinformatics prediction programs (TargetScan, miRanda, and DIANA) were used to identify putative target genes. E-cadherin was predicted as putative target of miR5003-3p and potential binding sequences were identified (Fig. 5E). E-cadherin has been shown to be a downstream target of miR-5003-3p in breast cancer [22], but whether it is regulated by miR-5003-3p in gastric cancer is unknown. As presented in Fig. 5E, we generated 2 luciferase reporters that contained the 3 putative binding sites. Luciferase reporter assays revealed that miR-5003-3p over-expression could reduce the activity of E-cadherin-2, but not E-cadherin-1 (Fig. 5F), suggesting that the miR-5003-3p-targeted sequences were contained in the E-cadherin-2 reporter. We then constructed 3 mutant reporters as presented in Fig. 5E, and these reporters were individually co-transfected with the miR-5003-3p mimic. Luciferase activity was found to be repressed in a single mutant reporter, but not in the double mutant

Fig. 4. Effects of miR-5003-3p on gastric cancer cell migration and invasion in vitro. A. Wound healing assays were performed to investigate migratory ability after miR-5003-3p down-expression. B. Wound healing assays were performed to investigate cell migration after miR-5003-3p up-regulation. C. Transwell assays were performed to identify changes in the migratory and invasive abilities of gastric cancer cells after transfection with in-miR-5003 or controls. D. Transwell assays were performed to identify changes in the migratory and invasive abilities of gastric cancer cells after transfection with miR-5003 or controls. E. E-cadherin immunostaining was weakly positive in gastric cancer tissues with relatively high miR5003-3p expression, while E-cadherin immunostaining was strong-


ly positive in gastric cancer tissues with low miR-5003-3p expression. ${ }^{*} \mathrm{P}<0.05$; ${ }^{*} \mathrm{P}<0.01$. 
Fig. 5. miR-5003-3p promotes EMT in gastric cancer cells by binding to E-cadherin. A. Relative mRNA levels of EMT pathway markers in MKN-1 cells after miR-50033 p knockdown. B. Relative mRNA levels of EMT pathway markers in SGC-7901 cells after miR-5003$3 p$ over-expression. C. Western blot analysis of EMT markers in MKN-1 cells transfected with inmiR-5003 or control. D. Western blot analysis of EMT markers in SGC-7901 cells transfected with miR-5003 or control. E. E-cadherin was predicted as a putative target of miR-5003-3p by bioinformatics prediction programs, and the wildtype and mutant binding sequences are presented. F. MKN-1 cells were transfected with in-miR-5003 or controls, and the activity of firefly and Renilla luciferase was measured. G. SGC-7901 cells were transfected with miR-5003 or controls, and the activity of firefly and Renilla luciferase was measured. The luciferase reporter assays showed that the 3' UTR of E-cadherin-2 containing the sequences $A$ and $B$ was targeted directly by miR-5003-3p. ${ }^{*} \mathrm{P}<0.05$; $* * \mathrm{P}<0.01$.

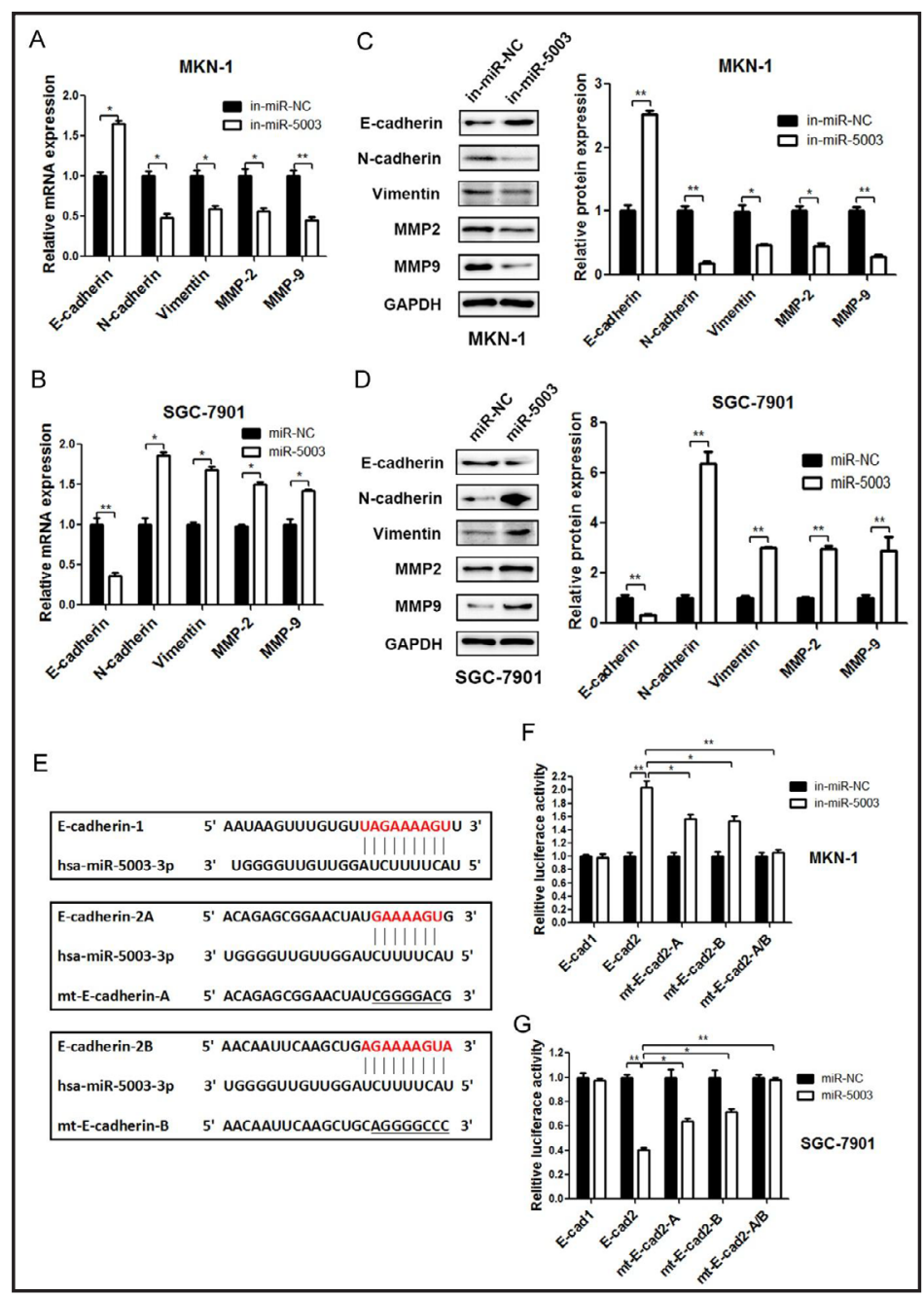

reporter, which confirmed that miR-5003-3p directly targeted the $3^{\prime}$ UTR of E-cadherin at sites A and B (Fig. 5). Another group of luciferase reporter assays indicated similar results (Fig. 5G). Taken together, we came to the conclusion that miR-5003-3p promoted migration, invasion, and EMT in gastric cancer by directly targeting E-cadherin.

Suppressive effects of RP11-789C1.1 on migration, invasion, and EMT in gastric cancer are mediated by miR-5003-3p/E-cadherin

To explore whether the effects of RP11-789C1.1 on migration, invasion, and EMT in gastric cancer were mediated by miR-5003-3p/E-cadherin, MKN-1 cells were co-transfected with pcDNA-RP and miR-5003, whereas siRNA-RP and in-miR-5003 were transfected into SGC-7901 cells. The detection of E-cadherin mRNA expression demonstrated that pcDNA-RP increased E-cadherin expression remarkably, which was repressed by miR-5003 (Fig. 6A). Conversely, there was a decrease of E-cadherin expression in siRNA-RP-transfected cells, which could be rescued by in-miR-5003 (Fig. 6B). Meanwhile, co-transfection of pcDNA-RP and miR-5003 clearly reversed the expression of miR-5003-3p compared with pcDNA-RP transfection alone (Fig. 6C). The detection of miR-5003-3p expression in SGC-7901 cells illustrated similar results (Fig. 6D). Transwell assays demonstrated that the anti-metastatic effects of RP11-789C1.1 over-expression on MKN-1 cell migration and invasion could be attenuated by miR-5003 (Fig. 6E). Conversely, miR-5003-3p knockdown repressed the migration and invasion of RP11-789C1.1-silenced SGC-7901 cells (Fig. 6F). In addition, the 
Fig. 6. miR-5003-3p mediates the tumor suppressive effects of RP11-789C1.1 in gastric cancer. A. miR-5003-3p restoration reduced the expression of E-cadherin in MKN-1 cells over-expressing RP11-789C1.1. B. miR-5003$3 \mathrm{p}$ knockdown increased the expression of E-cadherin in SGC7901 cells under-expressing RP11-789C1.1. C, D. Transfection efficiencies and miR-5003-3p levels after pcDNA-RP+miR-5003 and siRNA-RP+in-miR-5003 cotransfection were verified. E. Transwell assays showed that miR-5003-3p could largely reverse the suppressive effects of RP11789C1.1 on the migration and invasion of MKN-1 cells. F. miR5003-3p silencing abrogated the pro-metastatic effects of RP11789C1.1 knockdown in SGC-7901 cells. G. miR-5003-3p could largely reverse the suppressive effects of RP11-789C1.1 on the expression of E-cadherin and vimentin in MKN-1 cells. H. MiR-5003-3p knockdown could abolish the promoting effects of RP11-789C1.1 on E-cadherin and vimentin expression in SGC7901 cells. 1 = pcDNA-NC+miR-

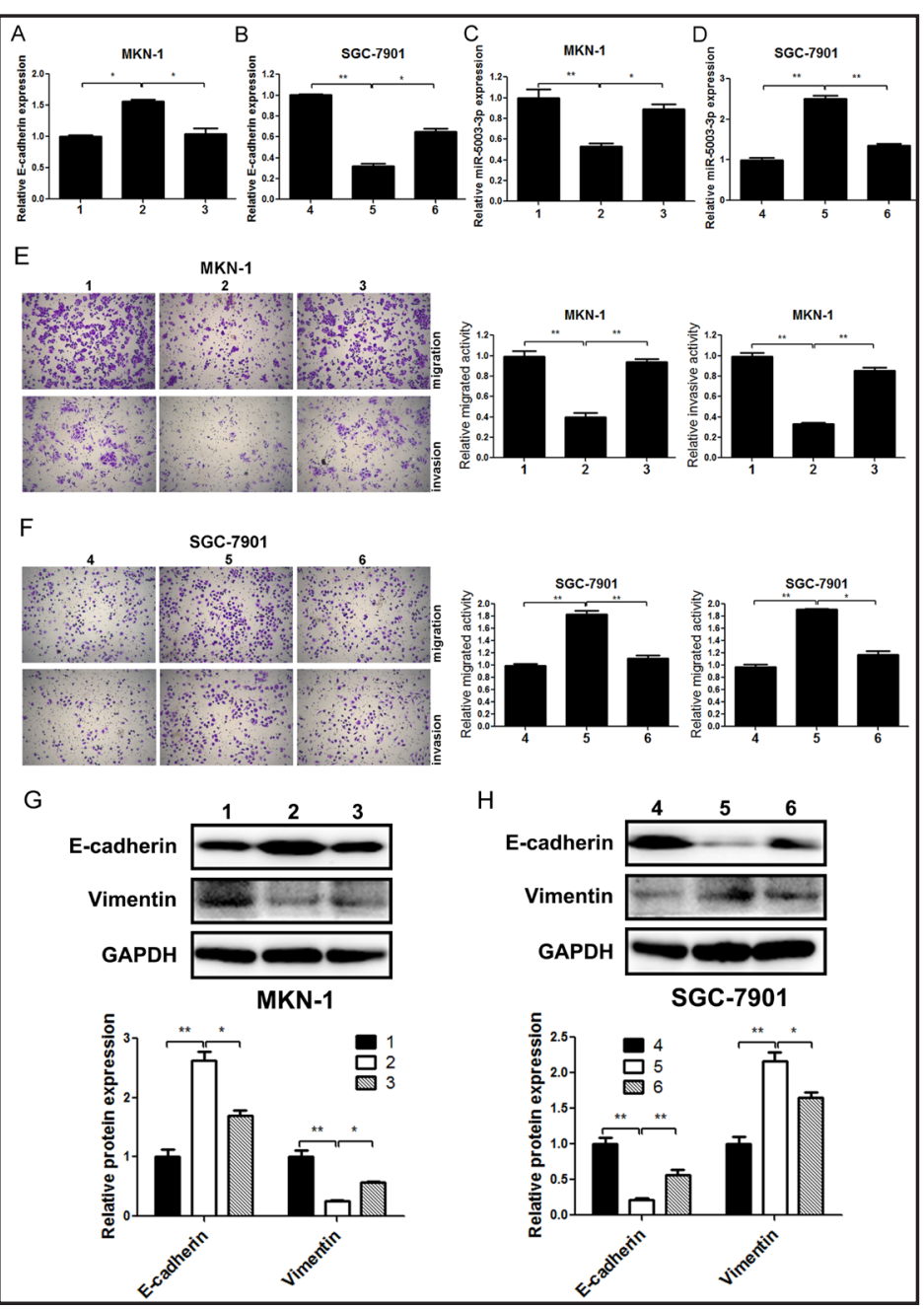
NC; 2 = pcDNA-RP+miR-NC; 3 = pcDNA-RP+miR-5003; 4 = siRNA-NC+in-miR-NC; 5 = siRNA-RP+in-miR-NC; 6 = siRNA-RP+in-miR-5003. ${ }^{*} \mathrm{P}<0.05 ;{ }^{* *} \mathrm{P}<0.01$.

stimulative effects of RP11-789C1.1 silencing on the promotion of EMT could be reversed by miR-5003-3p knockdown (Fig. 6H). Consistently, western blot analysis demonstrated that miR-5003-3p over-expression could dramatically reverse the suppressive effects of RP11789C1.1 on the progression of EMT in gastric cancer cells (Fig. 6G). Taken together, these results suggested that miR-5003-3p/E-cadherin mediated the suppressive effects of RP11789C1.1 on migration, invasion, and EMT in gastric cancer.

\section{Discussion}

Being important members of the non-coding RNA family, lncRNAs have been found to be associated with many physiological and pathological processes, such as cell growth, differentiation, development, and tumorigenesis [23-25]. For their role in tumorigenesis, the aberrant expression of IncRNAs has an impact not only on cancer cell proliferation and metastasis and as prognostic factors [26] but also on EMT, tumor drug resistance, and cancer stem cells $[27,28]$. RP11-789C1.1 was identified initially in our previous study as a specific lncRNA that was significantly downregulated in gastric cancer tissues when compared with adjacent normal tissues [19]. It was also shown that RP11-789C1.1 expression was lower 
in gastric cancer tissues with lymph node metastasis than in those with no lymph node metastasis. Moreover, patients expressing RP11-789C1.1 at a low level showed significantly worse survival than those with high RP11-789C1.1 expression. Hence, we speculated that RP11-789C1.1 may serve as a suppressor of gastric cancer.

In this study, we explored and analyzed the biological function of RP11-789C1.1 in gastric cancer cells. Consistent with our previous speculation, the results of the present study suggested that RP11-789C1.1 could significantly suppress gastric cancer cell growth, migration, and invasion. Some studies have shown that lncRNAs can inhibit the progression of gastric cancer. Zhao et al. found that lncRNA SNHG5 was significantly downregulated in gastric cancer and it suppressed gastric cancer cell proliferation and metastasis in vitro and in vivo by trapping MTA2 [29]. LncRNA AF147447 could repress gastric cancer cell proliferation and invasion via MUC2 and miR-34c [30]. To the best of our knowledge, this is the first study to illustrate the biological function of RP11-789C1.1 in cancer cells.

RP11-789C1.1 was differentially identified from metastatic gastric cancer tissues and non-metastatic tissues, so we focused on its role in metastasis. EMT is a vital process in the progression of cancer cell metastasis, and accepted biomarkers of EMT include the reduced expression of E-cadherin and enhanced expression of $\mathrm{N}$-cadherin and vimentin [31]. It has been revealed that lncRNAs, miRNAs, and EMT are closely related or associated with each other in a variety of tumor types [32]. We thus explored the expression of EMT markers in gastric cancer cells in which RP11-789C1.1 was over-expressed or knocked down, and found that RP11-789C1.1 could inhibit the progression of EMT. These results, interestingly, correlated with our previous study in which the upregulation of IncRNA XLOC-010235 in gastric cancer cells promoted EMT [20]. Both of these IncRNAs were identified with the same microarray and were validated using clinical specimens. Future studies should explore whether there is any connection or regulatory network between these lncRNAs in gastric cancer cells, which may help to strengthen our understanding of their biological roles in tumor progression.

LncRNAs can act as ceRNA sponges for miRNAs to regulate downstream genes and exert vital roles in physiological and pathological processes [33]. Herein, we firstly identified that RP11-789C1.1 could target miR-5003-3p directly to suppress the migration, invasion, and EMT of gastric cancer cells. miR-5003-3p was previously screened as an EMT-regulating miRNA that could induce the upregulation of Snail and enhance the formation of metastatic nodules in the lungs of mice. The present study revealed that miR-5003-3p promoted migration, invasion, and EMT in gastric cancer. Moreover, E-cadherin was also identified as a direct target of miR-5003-3p in gastric cancer. To the best of our knowledge, this study is also the first to verify the tumor-promoting role of miR-5003-3p in gastric cancer cells. Subsequently, whether RP11-789C1.1 could regulate the expression of E-cadherin and EMT by targeting miR-5003-3p was validated and the mutual adjustments between RP11789C1.1 and miR-5003-3p were verified.

In conclusion, our study suggested that lncRNA RP11-789C1.1 could inhibit gastric cancer cell migration, invasion, and EMT. Moreover, the antitumor function of RP11-789C1.1 was mediated by the RP11-789C1.1/miR-5003-3p/E-cadherin axis. Our study provides a new hypothesis for the study and treatment of gastric cancer and suggests that the RP11789C1.1/miR-5003-3p/E-cadherin axis may serve as a prospective therapeutic target for gastric cancer.

\section{Acknowledgements}

This work was supported by grants from the National Natural Science Foundation of China (No. 81401307), the Science and Technology Development Project of Guangzhou (201508020024), the Natural Science Foundation of Guangdong (2014A030313060), the Science and Technology Planning Project of Guangdong (2016A020215044), and the Support Plan for Major Project Cultivation and Emerging Subjects of Sun Yat-sen University (16YKJC13). 


\section{Cellular Physiology Cell Physiol Biochem 2018;47:2432-2444 \begin{tabular}{l|l} 
DOI: 10.1159/000491617 & Ond Biochemistry 2018 The Author(s). Published by S. Karger AG, Basel \\
Published online: July 09, 2018 & www.karger.com/cpb
\end{tabular}

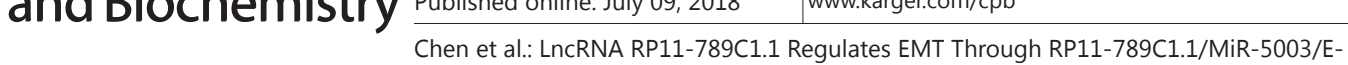 Cadherin Axis}

\section{Disclosure Statement}

The authors declare to have no competing interests.

\section{References}

$>1$ Siegel RL, Miller KD, Jemal A: Cancer Statistics, 2017 CA Cancer J Clin 2017;67:7-30.

-2 Patru CL, Surlin V, Georgescu I, Patru E: Current issues in gastric cancer epidemiology. Rev Med Chir Soc Med Nat Iasi 2013;117:199-204.

-3 Bartonicek N, Maag JL, Dinger ME: Long noncoding RNAs in cancer: mechanisms of action and technological advancements. Mol Cancer 2016;15:43.

4 Schmitz SU, Grote P, Herrmann BG: Mechanisms of long noncoding RNA function in development and disease. Cell Mol Life Sci 2016;73:2491-2509.

5 Naidu S, Magee P, Garofalo M: MiRNA-based therapeutic intervention of cancer. J Hematol Oncol 2015;8:68.

6 Li L, Geng Y, Feng R, Zhu Q, Miao B, Cao J, Fei S: The Human RNA Surveillance Factor UPF1 Modulates Gastric Cancer Progression by Targeting Long Non-Coding RNA MALAT1 Cell Physiol Biochem 2017;42:2194-2206.

7 Yan J, Zhang Y, She Q, Li X, Peng L, Wang X: Long Noncoding RNA H19/miR-675 Axis Promotes Gastric Cancer via FADD/Caspase 8/Caspase 3 Signaling Pathway. Cell Physiol Biochem 2017;42:2364-2376.

-8 Necsulea A, Soumillon M, Warnefors M, Liechti A, Daish T, Zeller U: The evolution of lncRNA repertoires and expression patterns in tetrapods. Nature 2014;505:635-640.

-9 Ambros V: The functions of animal microRNAs. Nature 2004;431:350-355.

10 Yan K, Tian J, Shi W, Xia H, Zhu Y: LncRNA SNHG6 is Associated with Poor Prognosis of Gastric Cancer and Promotes Cell Proliferation and EMT through Epigenetically Silencing p27 and Sponging miR-101-3p. Cell Physiol Biochem 2017;42:999-1012.

-11 Yu B, Ye X, Du Q Zhu B, Zhai Q Li XX: The Long Non-Coding RNA CRNDE Promotes Colorectal Carcinoma Progression by Competitively Binding miR-217 with TCF7L2 and Enhancing the Wnt/beta-Catenin Signaling Pathway. Cell Physiol Biochem 2017;41:2489-2502.

12 Mercer TR, Dinger ME, Mattick JS: Long non-coding RNAs: insights into functions. Nat Rev Genet 2009;10:155-159.

13 Nagano T, Fraser P: No-nonsense functions for long noncoding RNAs. Cell 2011; 145: 178-181.

14 Wang B, Yang H, Shen L, Wang J, Pu W, Chen Z: Rs56288038 (C/G) in 3'UTR of IRF-1 Regulated by MiR-5025p Promotes Gastric Cancer Development. Cell Physiol Biochem 2016;40:391-399.

15 Hou P, Zhao Y, Li Z, Yao R, Ma M, Gao Y: LincRNA-ROR induces epithelial-to-mesenchymal transition and contributes to breast cancer tumorigenesis and metastasis. Cell Death Dis 2014;5:e1287.

16 Han Y, Liu Y, Zhang H, Wang T, Diao R, Jiang Z: Hsa-miR-125b suppresses bladder cancer development by down-regulating oncogene SIRT7 and oncogenic long non-coding RNA MALAT1 FEBS Lett 2013;587:3875-3882.

17 Thiery JP, Acloque H, Huang RY, Nieto MA: Epithelial-mesenchymal transitions in development and disease. Cell 2009;139:871-890.

18 Perl AK, Wilgenbus P, Dahl U, Semb H, Christofori G: A causal role for E-cadherin in the transition from adenoma to carcinoma. Nature 1998;392:190-193.

19 Song W, Liu YY, Peng JJ, Liang HH, Chen HY, Chen JH: Identification of differentially expressed signatures of long non-coding RNAs associated with different metastatic potentials in gastric cancer. J Gastroenterol 2016;51:119-129.

20 Liu YY, Chen ZH, Peng JJ, Wu JL, Yuan YJ, Zhai ET: Up-regulation of long non-coding RNA XLOC_010235 regulates epithelial-to-mesenchymal transition to promote metastasis by associating with Snail1 in gastric cancer. Sci Rep 2017;7:2461.

21 Chen Z, Han S, Huang W, Wu J, Liu Y, Cai S: MicroRNA-215 suppresses cell proliferation, migration and invasion of colon cancer by repressing Yin-Yang 1 Biochem Biophys Res Commun 2016;479:482-488.

22 Kwak SY, Yoo JO, An HJ, Bae IH, Park MJ, Kim J: MiR-5003-3p promotes epithelial-mesenchymal transition in breast cancer cells through Snail stabilization and direct targeting of E-cadherin. J Mol Cell Biol 2016. 


\section{Cellular Physiology Cell Physiol Biochem 2018;47:2432-2444 \begin{tabular}{l|l} 
DOI: 10.1159/000491617 & $\begin{array}{l}\text { O 2018 The Author(s). Published by S. Karger AG, Basel } \\
\text { www.karger.com/cpb }\end{array}$
\end{tabular} \\ Chen et al.: LncRNA RP11-789C1.1 Regulates EMT Through RP11-789C1.1/MiR-5003/E- \\ Cadherin Axis}

23 Liang T, Zhou B, Shi L, Wang H, Chu Q Xu F: IncRNA AK017368 promotes proliferation and suppresses differentiation of myoblasts in skeletal muscle development by attenuating the function of miR-30c. FASEB J 2017;32:377-389.

24 Wang X, Li T, Li M, Cao N, Han J: The Functional SOCS3 RS115785973 Variant Regulated by MiR-4308 Promotes Gastric Cancer Development in Chinese Population. Cell Physiol Biochem 2016;38:1796-1802.

-25 Saha P, Verma S, Pathak RU, Mishra RK: Long Noncoding RNAs in Mammalian Development and Diseases. Adv Exp Med Biol 2017;1008:155-198.

-26 Abraham JM, Meltzer SJ: Long Noncoding RNAs in the Pathogenesis of Barrett's Esophagus and Esophageal Carcinoma. Gastroenterology 2017;153:27-34.

-27 Sun M, Kraus WL: From discovery to function: the expanding roles of long noncoding RNAs in physiology and disease. Endocr Rev 2015;36:25-64.

28 Heery R, Finn SP, Cuffe S, Gray SG: Long Non-Coding RNAs: Key Regulators of Epithelial-Mesenchymal Transition, Tumour Drug Resistance and Cancer Stem Cells. Cancers (Basel) 2017;9.

-29 Zhao L, Guo H, Zhou B, Feng J, Li Y, Han T: Long non-coding RNA SNHG5 suppresses gastric cancer progression by trapping MTA2 in the cytosol. Oncogene 2016;35:5770-5780.

-30 Zhou X, Chen H, Zhu L, Hao B, Zhang W, Hua J: Helicobacter pylori infection related long noncoding RNA (lncRNA) AF147447 inhibits gastric cancer proliferation and invasion by targeting MUC2 and up-regulating miR-34c. Oncotarget 2016;7:82770-82782.

-31 Sanchez-Tillo E, Liu Y, de Barrios O, Siles L, Fanlo L, Cuatrecasas M: EMT-activating transcription factors in cancer: beyond EMT and tumor invasiveness. Cell Mol Life Sci 2012;69:3429-3456.

-32 Cao MX, Jiang YP, Tang YL, Liang XH: The crosstalk between IncRNA and microRNA in cancer metastasis: orchestrating the epithelial-mesenchymal plasticity. Oncotarget 2017;8:12472-12483.

-33 Deng G, Sui G: Noncoding RNA in oncogenesis: a new era of identifying key players. Int J Mol Sci 2013;14:18319-18349. 